\title{
Predictions for Oxygen Supply Control to Enhance Population Stability of Engineered Production Strains
}

\author{
Amit Varma and Bernhard O. Palsson* \\ Department of Chemical Engineering, University of Michigan, Ann Arbor, \\ Michigan 48109
}

Received September 10, 1993/Accepted September 13, 1993

\begin{abstract}
The simultaneous growth and product formation in a microbial culture is an important feature of several laboratory, industrial, and environmental bioprocesses. Metabolic burden associated with product formation in these bioprocesses may lead to growth advantage of a nonproducing mutant leading to a loss of the producing population over time. A simple population dynamics model demonstrates the extreme sensitivity of population stability to the engineered productivity of a strain. Here we use flux balance analysis to estimate the effects of the metabolic burden associated with product secretion on optimal growth rates. Comparing the optimal growth rates of the producing and nonproducing strains under a given processing condition allows us to predict the population stability. In order to increase stability of an engineered strain, we determine processing conditions that simultaneously maximize the growth rate of the producing population while minimizing the growth rate of a nonproducing population. Using valine, tryptophan, and lysine production as specific examples, we demonstrate that although an appropriate choice of oxygenation may increase culture longevity more than twofold, total production as governed by economic criterion can be increased by several orders of magnitude. Choice of optimal nutrient and oxygen supply rates to enhance stability is important both for strain screening as well as for culture of engineered strains. Appropriate design of the culture environment can thus be used to enhance the productivity of bioprocesses that use engineered production strains. (c) 1994 John Wiley \& Sons, Inc.

Key words: Escherichia coli • amino acids - linear optimization - metabolic fluxes - metabolic engineering • culture stability • oxygen
\end{abstract}

\section{INTRODUCTION}

The biological route for the production of chemicals utilizes cellular metabolism to convert a substrate into the desired product. An important limitation of industrial biochemical production is the population instability of an engineered strain that results from overgrowth of a nonproducing mutant population. As reported in the literature, even a small growth advantage of nonproducers can result in their rapid outgrowth of the engineered strain (e.g., refs. 6, 8, 14). Engineering of cells to redirect metabolic fluxes toward the desired product ${ }^{2,23}$ produces an unnatural condition within the cell which may compromise stability of the engineered

\footnotetext{
* To whom all correspondence should be addressed
}

clone. It is thus desirable to provide culture conditions that favor growth of the engineered production strain.

Stability of recombinant cells in large-scale culture depends on strain, or genetic stability, as well as population stability. ${ }^{6}$ Strain stability as represented by the mutation of the engineered cells is determined by strain properties, the nature of engineered changes, and the genetic techniques used. Strain stability is therefore affected by choices of vector, copy number, etc., as well as process parameters such as temperature, $\mathrm{pH}$, nutrient concentration, etc. Although several methods can be used to reduce the mutational frequency, it is not possible to eliminate mutations in a large-scale industrial culture. ${ }^{4}$

Population instability, on the other hand, refers to the outgrowth of the engineered strain by a mutant strain, resulting from the growth advantage of a mutant population over the engineered strain. Population stability may be affected by the biochemical and physical variables that define the processing conditions. In the present study we take the first step toward providing a systemic metabolic basis for determining the influence of process conditions on the stability of a producing population.

We use a population balance analysis to determine the total productivity of a culture based on economic consideration of production. We determine the impact of engineered productivity (with the resulting loss in growth) on population stability and total production. Optimal metabolism and growth rates of both producing and nonproducing populations are determined by a flux balance model. Flux balance analysis has been used to determine the capabilities of microbial metabolism. ${ }^{3,9,20,27,29}$ This approach has been successfully applied to Escherichia coli metabolism to study optimal growth patterns ${ }^{28}$ as well as the production of biochemicals. ${ }^{25}$ Here we use flux balance analysis to investigate simultaneous growth and biochemical production to determine population stability under various oxygenation conditions.

Our hypothesis is that the influence of oxygenation on the growth rate would be different for the engineered producing strain and the nonproducing population of mutant strains. The rationale for this hypothesis is that different amounts of reductive power are required for generating biomass than for the product formation. Therefore, a change in the oxygen supply which is the redox sink would differentially 
affect the growth rate of producer and nonproducer cells. Computed productivity of valine, lysine, and tryptophan are used here to illustrate the enhancement in culture stability that can be achieved through a suitable control of the oxygen supply rate.

\section{METHODS}

\section{Flux Balance Analysis}

This method uses metabolic flux balances to define limits on metabolic capabilities. The steady-state flux balance equation is written as

$$
\mathbf{S} \cdot \mathbf{v}=\mathbf{b}
$$

where $\mathbf{S}$ is the stoichiometric matrix of the metabolic network, $\mathbf{v}$ is the vector of reaction fluxes, and $\mathbf{b}$ is the net output from cellular metabolism. The general methods of flux balance analysis are outlined in the literature. ${ }^{3,9,20,27,29}$

The stoichiometric matrix used in the present analysis consists of the catabolic and anabolic reactions and has been defined elsewhere. ${ }^{25,27}$ Equation (1) is underdetermined since the number of fluxes exceeds the number of metabolites. Thus, a plurality of solutions exists and a particular solution may be found using linear optimization by stating an objective and seeking its maximal value within the stoichiometrically constrained domain.

\section{Objective Function}

An objective function that describes maximal growth rate, Equation (2) was used in this study:

$$
\min Z=-v_{\text {gro }}
$$

where the biosynthetic growth flux $\left(v_{\text {gro }}\right)$ is defined as

$$
\sum_{\text {all } M} d_{M} \cdot M \stackrel{v_{\mathrm{gro}}}{\longrightarrow} \text { biomass }
$$

Metabolic requirements for growth, $d_{M}$ moles of metabolite $M$ per gram biomass, have been defined ${ }^{25}$ based on the chemical composition of $E$. coli. ${ }^{7,12}$ With an implicit basis of $1 \mathrm{~g} \mathrm{DW}$-h the growth flux, $v_{\mathrm{gro}}$, represents grams of biomass produced per gram dry weight per hour, which is the growth rate.

In addition to the composition-based metabolic demands for growth, there are additional maintenance requirements. Maintenance was specified as $23 \mathrm{mmol}$ adenosine triphosphate (ATP)/g biomass for growthassociated and $5.87 \mathrm{mmol} \mathrm{ATP} / \mathrm{g}$ DW-h for non-growthassociated maintenance in the flux balance model. ${ }^{10,13,21,26}$

A simplex implementation of linear optimization was used $^{5}$ to find the optimal solutions. The solution provides both the optimal growth rate as well as the flux distribution in the metabolic network.

\section{Shadow Prices}

The mathematical dual of the linear optimization problem ${ }^{11}$ is also evaluated. The dual variables $\left(\gamma_{i}\right)$, or the shadow prices, Eq. (4), provide useful measures of the value of a metabolic intermediate toward optimizing the objective $Z$ :

$$
\gamma_{i}=-\frac{\partial Z}{\partial b_{i}}
$$

Thus, the shadow price for the $i$ th metabolite represents the marginal increase in growth rate $(-\partial Z)$ due to additional amounts of the metabolite $\left(\partial b_{i}\right)$. The quantity $\gamma_{i}$ therefore represents the utility or usefulness of the $i$ th metabolite to accelerate the growth rate of the cell.

\section{Uptake and Secretion}

Oxygen consumption of $E$. coli is assumed to be limited to $20 \mathrm{mmol}_{2} / \mathrm{g}$ DW-h due to enzymatic constraints. ${ }^{1}$ Oxygen consumption can be lower than this value due to either reduced metabolic requirements or external mass transfer limitations. Variation in oxygenation from completely anaerobic conditions to full oxygenation can be achieved by specifying the oxygen supply rate in the metabolic network. The engineered and mutant strain may not have the same optimal oxygen utilization rate. The two strains are, however, growing under the same processing conditions. Thus, we permit oxygen consumption up to the level of the supply rate in order to satisfy the maximal growth objective.

The by-product secretion pathways for acetate, formate, ethanol, lactate, and succinate have been included in the metabolic network to allow for by-product secretion under partially aerobic conditions. ${ }^{9,22,26}$ The choice to secrete any of these by-products is determined by optimization of the stated maximal growth objective. In addition, although a glucose supply of $10 \mathrm{mmol} \mathrm{Glc} / \mathrm{g} \mathrm{DW}$-h has been specified for all the computations, the results can be appropriately scaled for other glucose supply rates based on the oxygento-glucose supply ratio.

\section{Analysis of Population Stability}

Established differential equations, Eqs. (5) and (6), for describing the dynamics of population stability of producer and nonproducer clones ${ }^{6,8,14}$ were used in the present analysis:

$$
\begin{aligned}
& d x_{p} / d t=\mu_{p} x_{p}-\alpha x_{p} \\
& d x_{n} / d t=\mu_{n} x_{n}+\alpha x_{p}
\end{aligned}
$$

where $x$ is the cell number, the subscript $p$ refers to the population of producing cells and $n$ to the population of nonproducing cells, $\mu$ is the growth rate, and $\alpha$ is the mutational rate of conversion of producers to nonproducers. The dimensionless solution for the time-varying population dynamics is available $6,8,14$ :

$$
X_{p}(\tau)=e^{(1-A) \tau}
$$

$$
X_{n}(\tau)=\left[\frac{A}{1-A-S}\right]\left[e^{(1-A) \tau}-e^{S_{t}}\right]+X_{n 0} e^{S_{\tau}}
$$


where $\tau=\mu_{p} t$ is dimensionless time, $A=\alpha / \mu_{p}=$ $a / \ln ^{2}, a$ is the mutational probability per cell division, $S=\mu_{n} / \mu_{p}$ is the growth advantage of the nonproducing strain, and $X_{n 0}$ is the dimensionless initial nonproducer cell number.

It should be noted that the above and the following analysis does not include the potential influence of oxygen mass transfer limitations or the buildup of toxic by-products.

\section{Biochemical Production}

The flux balance analysis with a growth rate maximization objective has been shown to simulate the growth and metabolic behavior of $E$. coli. ${ }^{9,26-28}$ We can therefore use the flux balance model to determine the growth advantage of nonproducers over the producing population $\left(S=\mu_{n} / \mu_{p}\right)$.

Starting a culture with an engineered conversion, $C$ (mol product/mol substrate), the generation of nonproducers results in a reduction of net conversion. The net productivity at any period of time can be estimated as $C X_{p} /\left(X_{p}+X_{n}\right)$. An economic criterion for optimal culture termination in an industrial biochemical production can be formulated as a critical economic conversion. Thus, the decision to terminate the culture should occur when the net conversion falls below the predetermined threshold value based on the cost economics of production. Mathematically, the critical economic criteria may be stated as

$$
Y<\frac{C X_{p}}{X_{p}+X_{n}}
$$

where $Y$ is the critical conversion. The above result can be expressed in a nondimensional form, Eq. (10), by defining a dimensionless engineered conversion as $P=C / C_{\max }$ and a dimensionless economic conversion as $\sigma=Y / C_{\max }$ :

$$
\frac{\sigma}{P}<\frac{X_{p}}{X_{p}+X_{n}}
$$

Here $C_{\max }$ represents the maximal theoretical conversion of substrate into the desired product. Since all the substrate is utilized to make the product, the growth rate is zero during a $C_{\max }$ product formation rate. For the products considered here the values of $C_{\max }$ are valine, 1 ; lysine, 0.78 ; and tryptophan, 0.41 (mol $/ \mathrm{mol}){ }^{25}$

\section{Assuming Linear Trade-Off between Growth Rate and Product Formation}

Some general results for designing specific productivity in strains can be derived by assuming a linear reduction in growth rate due to biochemical production in order to estimate the growth advantage, ${ }^{25} \mathrm{Eq}$. (11). It should be pointed out that genetic engineering for metabolite overproduction can result in several deleterious side-effects that can reduce the performance of the strain. In this sense Eq. (11) is an idealization and forms a goal for strain development.

$$
\mu_{p}=\mu_{n}\left(1-\frac{C}{C_{\max }}\right)
$$

or in a dimensionless form,

$$
S=\frac{1}{1-P}
$$

Based on the above economic consideration, one can estimate the time for culture termination and hence the final producer cell number from Eqs. (7) and (8). A time integral of product formation up to the termination of the culture yields an estimate of the total production (TP), Eqs. (13) and (14). The total production in terms of dimensionless parameters (assuming $A<<1$ ) is given by Eq. (15):

$$
\begin{aligned}
T P & =\int_{0}^{t_{f}} r_{s} \cdot C \cdot X_{p} d t \\
& =\frac{r_{s} \cdot C}{(1-A) \mu_{p}}\left[e^{(1-A) \mu_{p} t_{f}}-1\right] \\
& =\frac{r_{s} \cdot C_{\max }}{\mu_{n}} \frac{P}{1-P}\left(X_{p f}-1\right) \quad \text { if } A<<1
\end{aligned}
$$

where the culture termination time $t_{f}$ and final cell number $X_{p f}$ are determined from the above economic considerations, Eq. (10). From an operational standpoint, the total production determined here is based on the assumption of operation in an exponential phase. Physical capacity limits of the equipment that result in an earlier culture termination are not considered here.

\section{RESULTS}

\section{Culture Stability}

Flux balance analysis allows the computation of the optimal growth rate which is a good approximation to experimental observations., ${ }^{9,26}$ Reduction in the optimal growth rate due to product secretion can also be estimated from the flux balance model. Thus, we can determine culture stability as measured by the growth advantage of nonproducers.

Let us first consider changes in culture stability for some illustrative producers of histidine, arginine, lysine, valine, and ethanol as a function of the oxygen supply. The growth advantage $(S)$, determined using the flux balance model, as a function of the oxygen supply for a fixed glucose supply rate of $10 \mathrm{mmol} \mathrm{Glc/g} \mathrm{DW-h}$ is shown in Figure 1. For some secreted products, the growth advantage has a minimum or optimal value at a particular oxygen supply rate (Fig. 1). A lower growth advantage is desirable since it results in an increased longevity of the culture before outgrowth by a nonproducing population. Operating at an oxygen supply corresponding to the minimum growth advantage can therefore result in an increased productivity from the culture over time.

The optimal oxygen supply rate is determined by the nature of the specific product and in particular by the redox requirements for product formation. For illustration, one may consider the simple example of ethanol production. Since ethanol formation has the largest redox requirements, an ethanol-producing population is most stable at low oxygen supply rates, as evidenced by the minimum growth advantage (Fig. 1). However, most products have a 


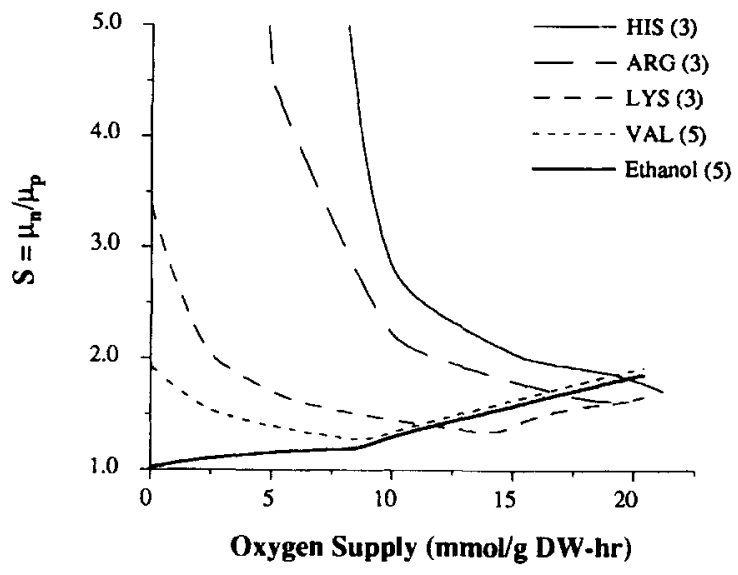

Figure 1. Culture stability represented by the growth advantage as a function of the oxygen supply for several biochemical products. A glucose supply rate of $10 \mathrm{mmol} \mathrm{Glc} / \mathrm{g} \mathrm{DW}$-h has been specified. The biochemical production (mmol $/ \mathrm{g} \mathrm{DW}-\mathrm{h}$ ) is listed in parentheses for the respective plots.

complex relationship between the growth advantage and the oxygen supply rate as defined by the metabolic network.

\section{Example: Valine Production}

To further define the effects of variation in the oxygen supply rate, we now focus our attention on valine production. Figure 2 shows the variation in the growth advantage $S$ as a function of the oxygen supply during valine production. A minimum growth advantage is found for all production rates of valine.

For oxygen supply below the optimum, the growth rate of both producing and nonproducing cells is similarly reduced. Because the growth rate of producer cells is lower, the percentage drop in their growth rate with reduced oxygen

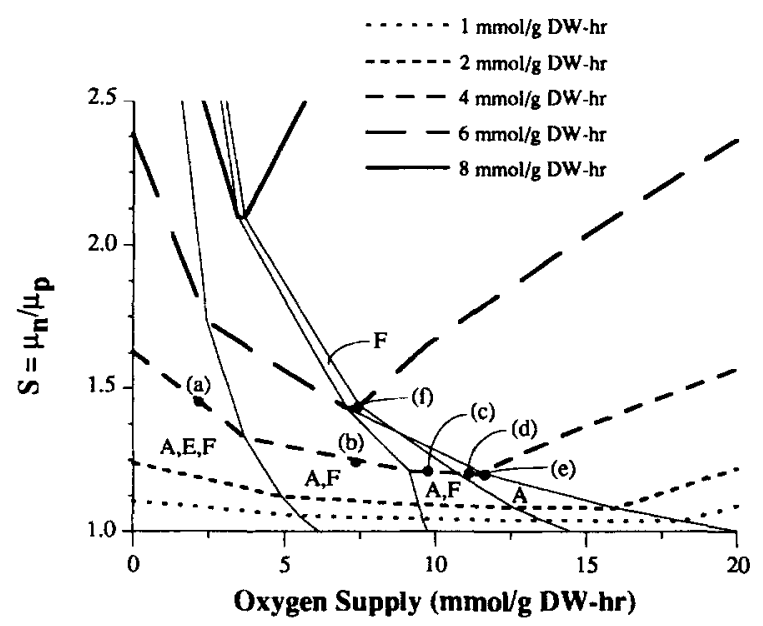

Figure 2. Culture stability during valine production as a function of the oxygen supply. The theoretical maximum valine production is $10 \mathrm{mmol}$ VAL $/ \mathrm{g} \mathrm{DW}$ - $\mathrm{h}$. Production rates are indicated in units of $\mathrm{mmol} / \mathrm{g} \mathrm{DW}-\mathrm{h}$. By-product secretion from the producer cells is shown in regions bounded by thin lines. Notation for secreted by-products is: A, acetate; E, ethanol; $\mathrm{F}$, formate. The flux distributions shown in Fig. 4 are determined at the conditions indicated by the lowercase letter in parentheses. supply is much greater, leading to high numerical values of the growth advantage $S$ at low oxygen supply rates. If oxygen supply is greater than the optimal value, we find that the producer cells are not affected since they have an adequate oxygen supply. They are unable to utilize the additional oxygen availability to enhance their growth rate. Hence we obtain a higher numerical value of the growth advantage at an oxygen supply higher than the optimal value.

The changes in the growth rates of producer and nonproducer populations with the oxygen supply are shown in Figure 3. While an increased oxygen supply results in a continuously increasing nonproducer growth rate, the producer population reaches a maximum growth rate at an oxygen supply of $7.5 \mathrm{mmol} \mathrm{O}_{2} / \mathrm{g} \mathrm{DW}-\mathrm{h}$. Thus, the growth advantage increases with oxygen supply at high oxygenation. At low oxygenation levels, although both growth rates increase with oxygen supply, the percentage effect is greater for the producing population. Thus, the growth advantage falls with increasing oxygen supply at lower oxygen supply rates.

The by-product secretion patterns during optimal growth and valine production are indicated in Figure 2. Catabolic flux distributions characteristic of these phases are shown in Figure 4. Under oxygen limitations we observe a change in the optimal metabolic pathways and the secretion of acetate, formate, and ethanol as a means to eliminate surplus redox. Although the tricarboxylic acid (TCA) cycle is active at high oxygenation (Figs. 4d,e), it is not operative during oxygen limitations. Also, as oxygenation increases (Figs. 4a-e), biosynthetic redox (NADPH) generation shifts from transhydrogenation to the pentose phosphate cycle. Thus, the different phases of byproduct secretion (Fig. 2) are characterized by changes in the optimal metabolic pathways.

By-product secretion under different oxygenations can also be explained by examining shadow prices for the various metabolites. Table I lists the shadow prices for various redox carriers, energy, and by-products typical of the different phases (Fig. 2). Once again the shadow price reflects the utility of a metabolite to accelerate growth. Thus, a negative value for the shadow price in Table I

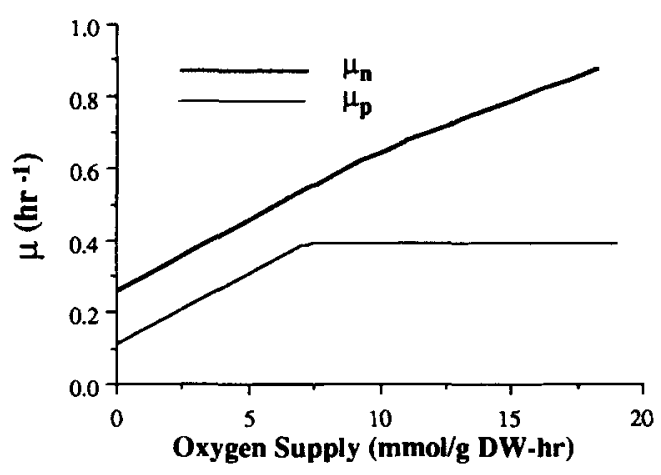

Figure 3. Variation of the growth rate of nonproducers and valine producers $(6 \mathrm{mmol} / \mathrm{g} \mathrm{DW}-\mathrm{h})$ as a function of the oxygen supply. 

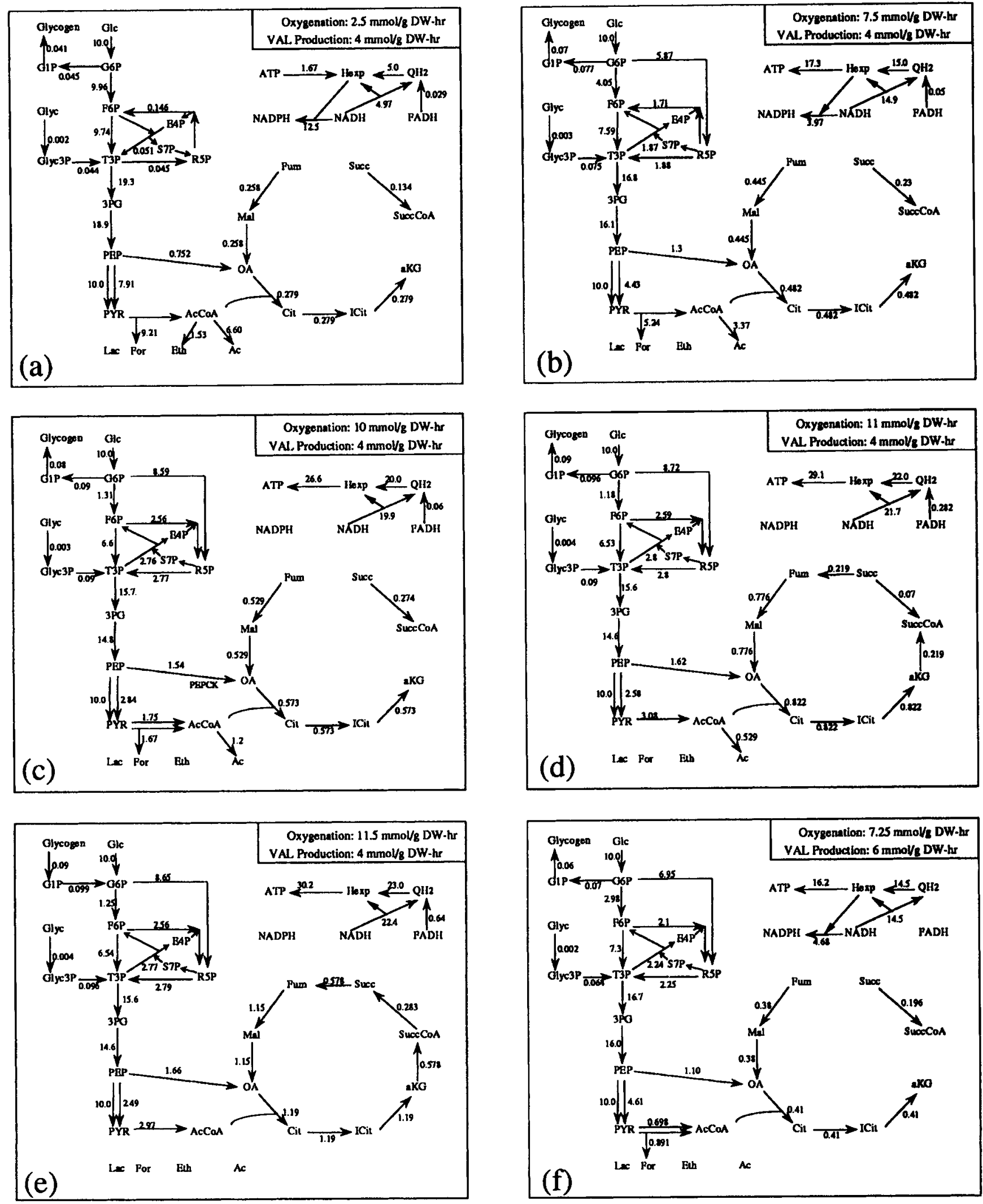

Figure 4. Flux distributions for the various phases of growth during valine production observed in Fig. 2 . A glucose supply of $10 \mathrm{mmol}$ Glc/g DW-h is specified and other parameters are listed in Table $\mathbf{I}$. 
Table I. Shadow prices of redox, energy, and by-products in the various phases of growth and by-product secretion observed in Figure 2 .

\begin{tabular}{|c|c|c|c|c|c|c|}
\hline & \multicolumn{6}{|c|}{ Flux distributions in Figure 2} \\
\hline & (a) & (b) & (c) & (d) & (e) & (f) \\
\hline \multirow{2}{*}{\multicolumn{7}{|c|}{$\begin{array}{l}\text { Production rate } \\
\mathrm{mmol} / \mathrm{g} \mathrm{DW} \cdot \mathrm{hr}\end{array}$}} \\
\hline & 4 & 4 & 4 & 4 & 4 & 6 \\
\hline $\begin{array}{l}\text { Oxygen supply } \\
\mathrm{mmol} / \mathrm{g} \mathrm{DW} \cdot \mathrm{hr}\end{array}$ & 2.5 & 7.5 & 10 & 11 & 11.5 & 7.25 \\
\hline$\mu(1 / \mathrm{hr})$ & 0.26 & 0.45 & 0.54 & 0.57 & 0.58 & 0.39 \\
\hline Metabolite & \multicolumn{6}{|c|}{ Shadow prices: $\partial \mu / \partial$ metabolite $\times 100$} \\
\hline NADPH & 0.183 & 0.289 & 0.359 & 0.389 & 0.808 & 0.522 \\
\hline NADH & -0.55 & -0.433 & 0 & 0.106 & 0.673 & 0 \\
\hline $\mathrm{QH}_{2}$ & -1.28 & -1.15 & -0.719 & -0.602 & 0.337 & -0.522 \\
\hline $\mathrm{O}_{2}$ & 4.03 & 3.75 & 2.88 & 2.62 & 0 & 2.09 \\
\hline $\mathrm{Hexp}^{a}$ & 0.367 & 0.361 & 0.359 & 0.354 & 0.168 & 0.261 \\
\hline Acetate & 0 & 0 & 0 & 0 & 2.49 & 1.57 \\
\hline Formate & 0 & 0 & 0 & 0.106 & 0.673 & 0 \\
\hline Ethanol & 0 & 0.216 & 1.08 & 1.27 & 4.34 & 2.35 \\
\hline Lactate & 0.55 & 0.649 & 1.08 & 1.27 & 4.34 & 2.35 \\
\hline Succinate & 0.367 & 0.577 & 1.44 & 1.73 & 5.18 & 2.61 \\
\hline
\end{tabular}

A glucose supply of $10 \mathrm{mmol} \mathrm{Glc} / \mathrm{g} \mathrm{DW} \cdot \mathrm{hr}$ is specified; valine production rate, and oxygen supply rate for the different phases are as listed.

${ }^{a}$ Hexp represents the energy of exporting one proton across the membrane. $\mathrm{QH}_{2}$ represents the hydrogenated form of quinone.

represents the undesirability of a metabolite. By-products that are undesirable are secreted and hence have a zero shadow price.

The different redox carriers are positioned in the respiratory chain in a hierarchical manner such that they have different energy values. This fact is clearly brought out by the differences in their shadow prices which represents their value to the cell. Thus, the value of redox is ordered as $\mathrm{NADPH}>\mathrm{NADH}>\mathrm{QH}_{2}$. The various phases are characterized by the value of the redox shadow prices as well as the by-product shadow prices. As oxygen supply becomes limiting, Table $\mathrm{I}(\mathrm{e}) \longrightarrow(\mathrm{a})$, we observe a reduction in the value of redox (lower shadow price). As a result, by-products are secreted in order to eliminate surplus redox. Under extreme oxygen limitations, Table I(a), the three by-products acetate, formate, and ethanol are secreted.

Thus, shadow prices as well as the optimal flux distributions show a metabolic pathway utilization characteristic of the various phases. These optimal flux distributions represent the goals of metabolic engineering to redirect catabolic fluxes toward the desired product.

\section{Engineering Optimal Conversion}

We now consider the impact of loss in productivity due to overgrowth of nonproducing cells. An optimal economic threshold for culture termination occurs when the net productivity of the culture falls below a predetermined marginal conversion based on the cost economics of production, Eq. (10).
As an example consider the total valine production possible, under the economic constraints outlined above, using cells of different engineered productivities (Fig. 5). The arbitrarily chosen economic threshold conversion of $0.4 \mathrm{~mol}$ valine/mol glucose denotes the conversion above which production is profitable. Thus, all strains engineered for a conversion greater than $0.4 \mathrm{~mol} / \mathrm{mol}$ are capable of an economically viable production.

A comparison of full oxygen supply to the optimal oxygen supply for culture stability shows that the total production can be increased by several orders of magnitude by a suitable control of oxygen supply (Fig. 5). Thus, optimal strain engineering as well as the selection of optimal culture conditions can have a tremendous impact on the production attainable in cultures. A suitable control strategy to implement these optimal culture conditions would have to be maintained both in a preculture as well as the production fermenter.

Figure 6 displays the average productivity of a culture as a function of time. It is evident from Figure 6 that control of oxygenation can have a tremendous impact on stabilizing the productivity. Thus, optimal oxygenation provides a longer economic process time for both cell growth and valine production, resulting in the larger total production. Indeed, an economic production time of $76 \mathrm{~h}$ implies a potential cell expansion of $5 \times 10^{11}$. A possible production time and cell expansion of this magnitude essentially implies that stability is not a problem.

In contrast, the engineered productivity has a smaller impact on the time for culture termination (Fig. 6). However, cell concentration reaches large values only toward the end 


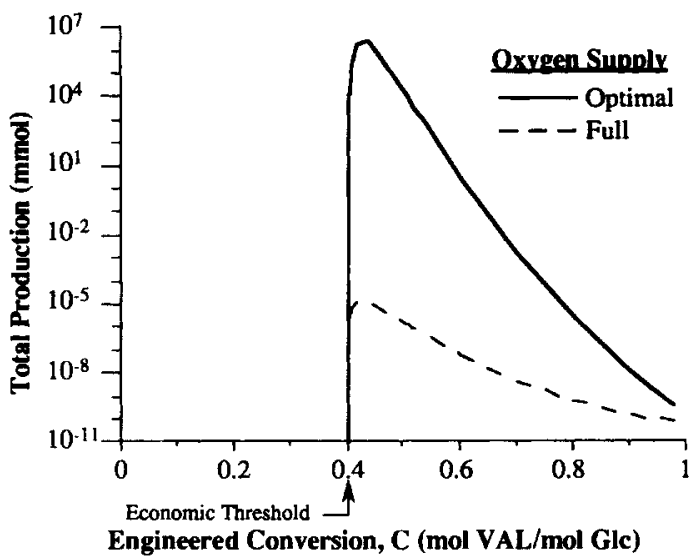

Figure 5. Total valine production in a culture as a function of the engineered specific productivity under the economic constraints that the marginal conversion should not fall below $0.4 \mathrm{~mol} \mathrm{VAL} / \mathrm{mol}$ Glc. Mutational rate $a=10^{-6}$ per cell division was assumed. The total production was calculated from Eq. (15).

of the culture, at which time most of the production occurs. Therefore, even the small difference in time causes a large change in the total production achievable, as evidenced in Figure 5. Maximal total production is observed to occur at an engineered productivity very close to the economic threshold conversion. Engineering productivity above this optimal value is counterproductive since there is an extremely rapid decay of the total production.

\section{Example: Tryptophan and Lysine}

To further demonstrate the principle of oxygenation control as a means to enhance culture productivity, we consider the production of the commercially important amino acids tryptophan and lysine. The culture stability parameter $S$ is shown at various production levels for these amino acids under different oxygenation conditions (Fig. 7). Once again

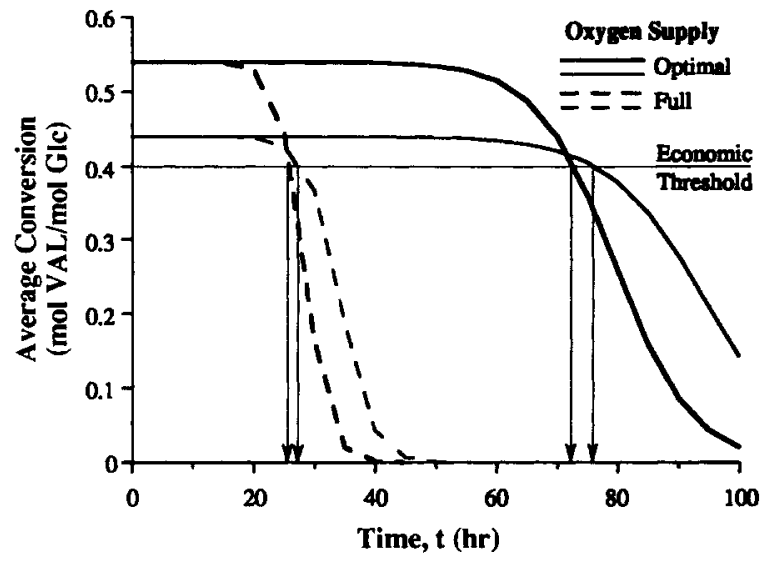

Figure 6. Average specific conversion of cultures computed as a function of time. The cultures start with single engineered clones producing 0.44 and $0.54 \mathrm{~mol} \mathrm{VAL} / \mathrm{mol} \mathrm{Glc}$. Optimal oxygenation conditions are shown to have a large impact on the production stability of the culture. Mutational rate $a=10^{-6}$ per cell division has been assumed. The termination times are indicated by arrows on the $x$ axis. we note that a reduced oxygen supply can result in an increased population stability of the producer cells.

Production levels achievable for the two amino acids are shown in Figure 8 based on the arbitrary economic criteria shown. Again optimal oxygenation is capable of enhancing productivity over several orders of magnitude. Furthermore, an optimal strain engineering strategy requires that specific productivity be engineered close to the economic threshold conversion in order to maximize total production.

From a closer look at Figure $8 \mathrm{~b}$ we note that a total production of $10^{13.5} \mathrm{mmol}$ lysine is possible under appropriate conditions. The high value of economic production possible reflects the fact that under these conditions stability does not restrict either the fermentation size or duration. In such a situation one may consider engineering for a higher level of substrate conversion to product.

\section{General Principles}

Independent of the choice of product some general principles of culture stability may be derived from the analysis just presented. Synthesis of any product results in the diversion of substrate from producing growth. Here we assume that a practically linear reduction in growth rate
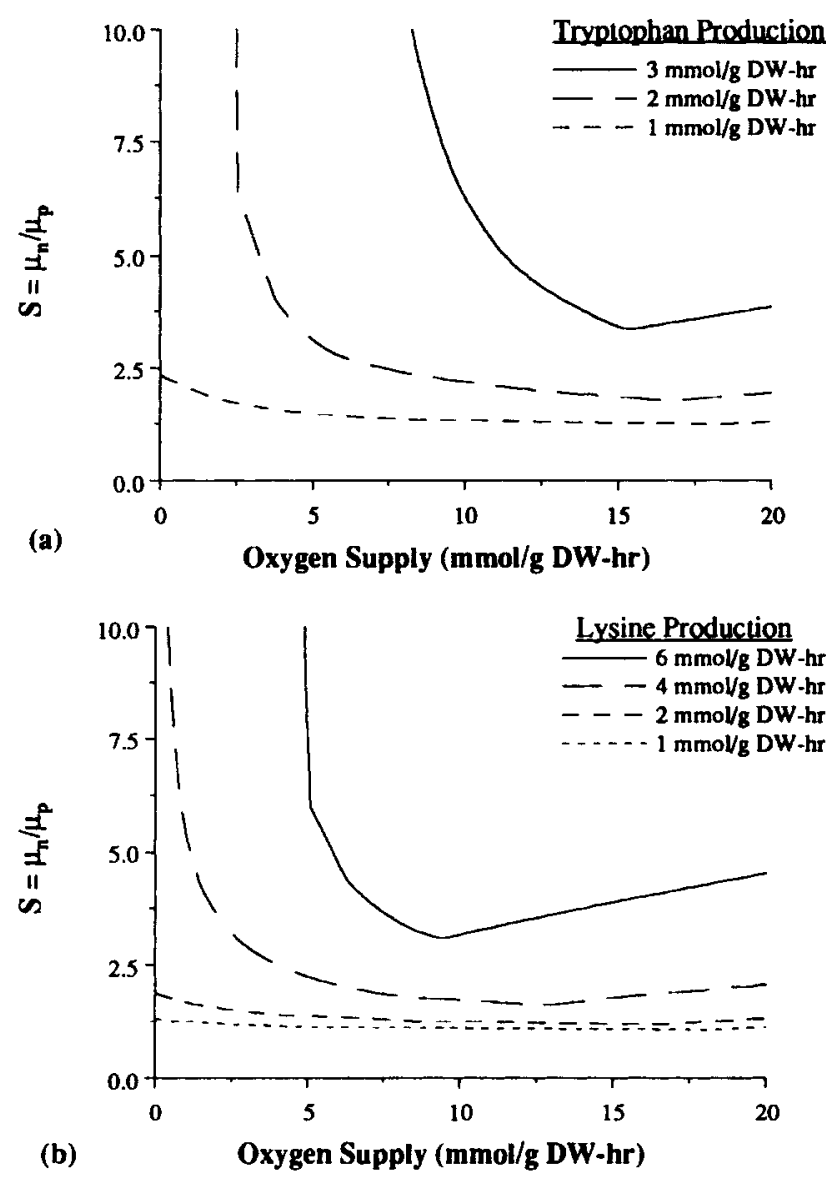

Figure 7. Culture stability represented by $S$ given as a function of the oxygen supply to the cells for (a) tryptophan and (b) lysine production. A glucose supply of $10 \mathrm{~mol} \mathrm{Glc/g} \mathrm{DW-h}$ was used for the computations. 


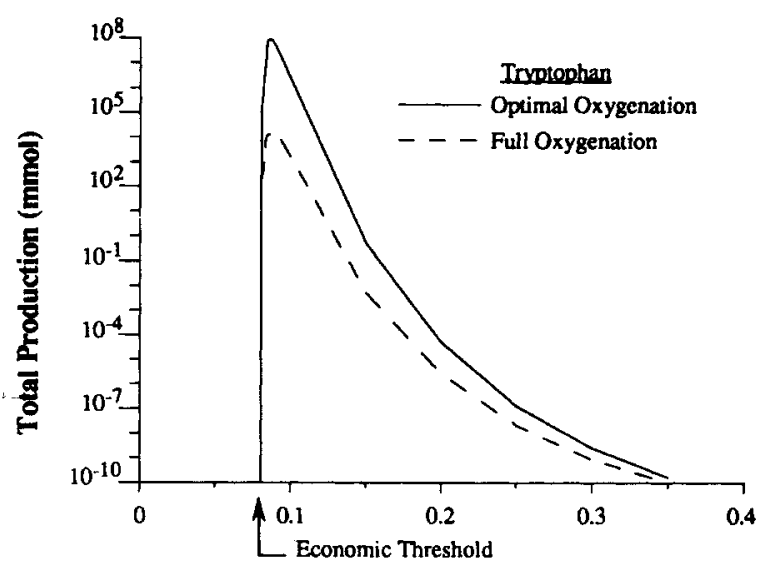

(a)

Engineered Conversion, C (mol TRP/mol Glc)

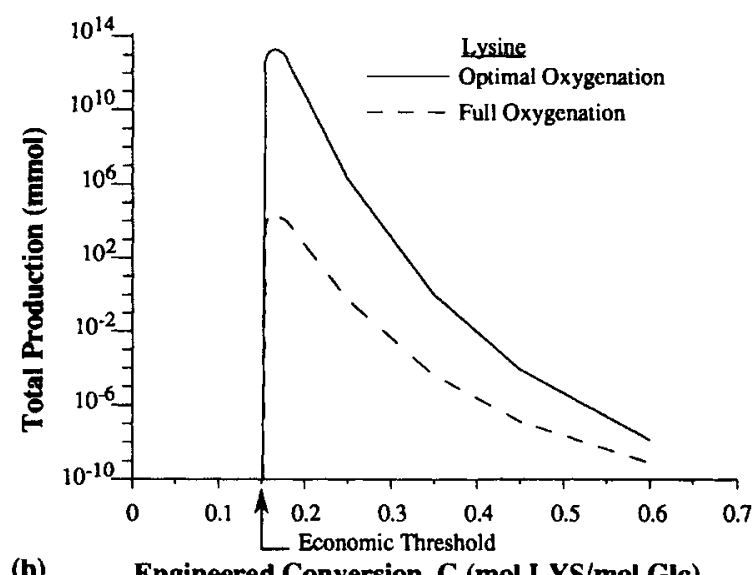

(b)

Engineered Conversion, C (mol LYS/mol Glc)

Figure 8. Total productivity of a culture is shown as a function of the specific productivity of the engineered clone with a glucose supply of $10 \mathrm{mmol} \mathrm{Glc/g} \mathrm{DW-h} \mathrm{for} \mathrm{(a)} \mathrm{tryptophan} \mathrm{and} \mathrm{(b)} \mathrm{lysine} \mathrm{production.} \mathrm{The}$ criterion for termination of the culture is that the marginal productivity falls below $0.8 \mathrm{mmol} \mathrm{TRP} / \mathrm{g} \mathrm{DW}$-h for tryptophan and $1.5 \mathrm{mmol} \mathrm{LYS} / \mathrm{g}$ $\mathrm{DW}$-h for lysine. Mutational rate $a=10^{-6}$ per cell division was assumed.

can be obtained during product secretion, ${ }^{25}$ as expressed by Eq. (11). Thus, the reduction in growth rate or the growth advantage can be estimated from the engineered productivity.

With the above estimation of the growth advantage we use population dynamics to estimate the culture size. Figure 9 shows the producer population size achievable before $25 \%$ of the total population converts to nonproducers. The probability of mutation, $a$, is seen to have a large influence on the culture size. Furthermore, the culture size decays rapidly with increasing engineered productivity. Thus, at a high productivity a reasonable producer population size can only be obtained by the use of selective conditions.

We next consider the total production possible under various conditions of engineered productivity and economic criteria. Figure 10 displays this relationship using the dimensionless parameters described in the methods section. Given that the production costs and economics of the market specify the minimum threshold conversion, we find

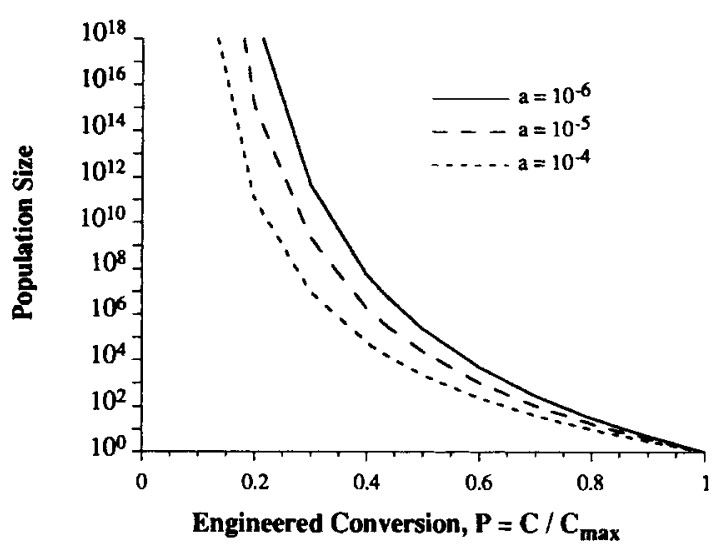

Figure 9. Culture size, as the number of producing cells obtainable, for the culture to become $25 \%$ nonproducing plotted as a function of the dimensionless engineered productivity. The mutation probability $(a)$ used in the computations is indicated.

that the maximal total production is very sensitive to the mutational probability $a$. We also find that optimal strain engineering calls for an engineered production rate close to the economic criteria. Indeed overengineering productivity to any extent may be highly undesirable, as seen by the rapid drop in total production.

The total production is observed to be infinite at the maximal dimensionless engineered productivity of unity $(P=1)$ (Fig. 10). At this maximal production the growth rate is reduced to zero, which eliminates the problems of culture stability which are growth related. Thus, the infinite production possible without growth corresponds to an alternative production technology of separating the growth and production phases.

\section{DISCUSSION AND CONCLUSIONS}

An important limitation of large-scale biochemical production is the population instability of the engineered cells due to mutations and overgrowth by nonproducing populations. Loss of productivity in the culture sets an economic limit on culture longevity of culture size. Laboratory strain screening with nonselective or poorly selective media can potentially suffer from outgrowth by low production mutant populations. In order to alleviate such problems of population stability, it is useful to identify the conditions favoring stability of a culture.

Several studies ${ }^{6,8,14}$ have utilized population balances to study the dynamics of different populations in a culture. Nonproducing populations are assumed to arise from the original clone by random mutations. Stability of the producer cells is found to be critically dependent on the growth advantage of nonproducers over the producer cells. Figure 11, derived from standard population balance models in the literature, ${ }^{6,8,14}$ shows the extreme sensitivity of culture size to the growth advantage of nonproducers over the original clone. An increase in the number of generations $\left(n_{p}\right)$ results in an exponential increase in the culture size as well as productivity. Thus, there is a significant incentive 

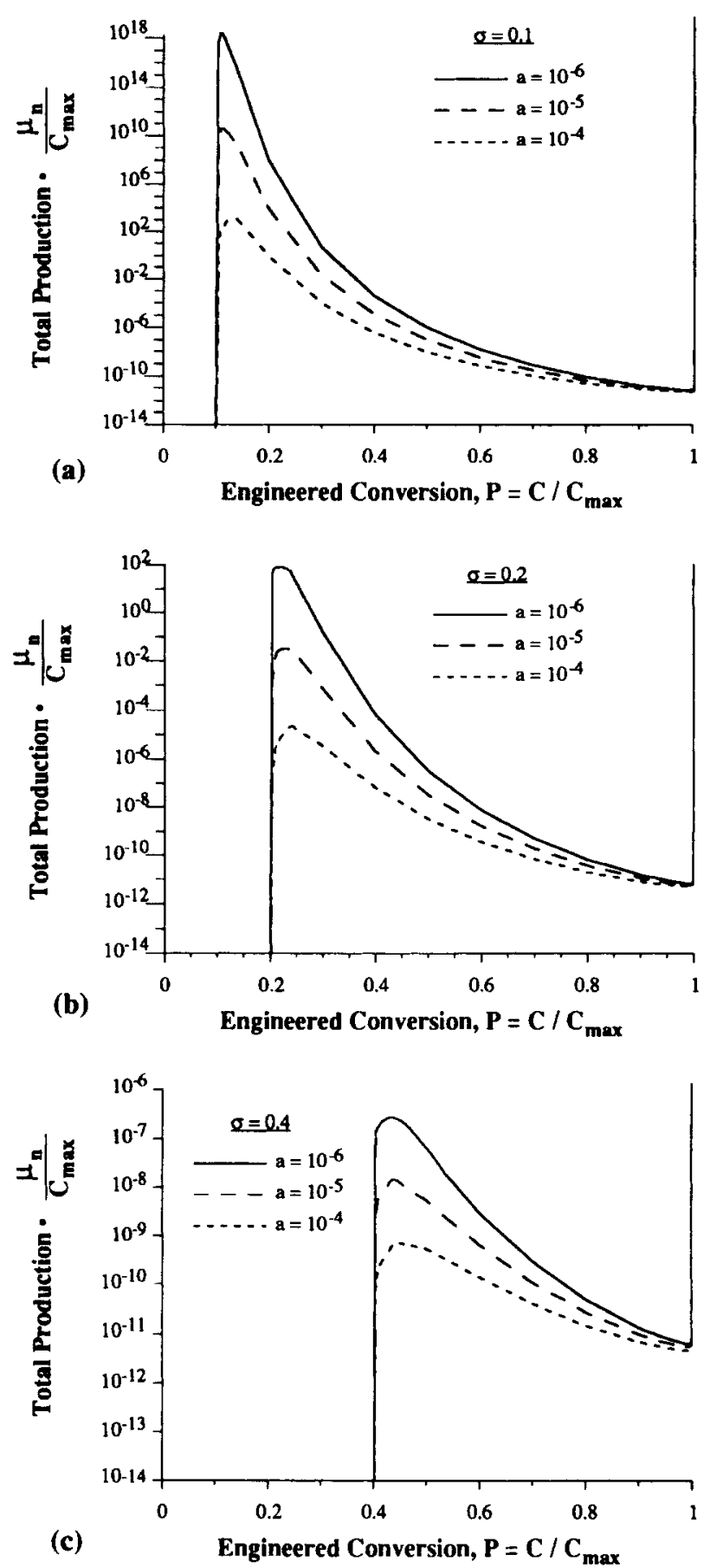

Figure 10. Total production of a biochemical product as a function of the dimensionless engineered productivity under different mutational probabilities and dimensionless economic criteria.

to determine conditions favoring culture stability for largescale industrial cultures.

On a single-cell level instability arises from a loss or mutation of genetic material that leads to a loss in productivity. This process has been characterized as strain or genetic instability and may arise from the segregational loss of extrachromosomal material, mutational loss of genes, or reversion of the genetic changes. Segregational losses can be substantially reduced by the use of selective techniques

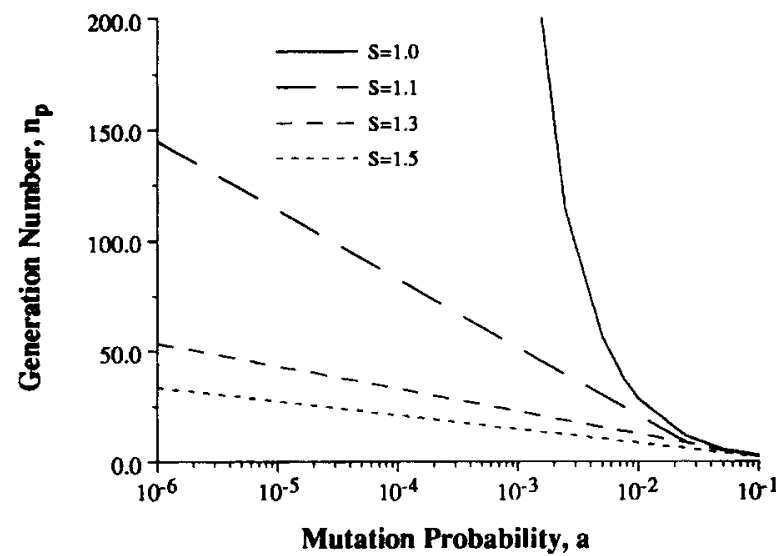

Figure 11. Stability of an engineered clone in the presence of mutations. The number of generations of the engineered producer strain $n_{p}$ for the culture conversion to $25 \%$ mutants is plotted as a function of the mutation probability $a$. Culture stability as measured by the number of generations is extremely sensitive to the growth advantage of the mutant shown as $S=\left(\mu_{n} / \mu_{p}\right)$

or a suitable choice of vector. However, random mutations in $E$. coli genetic material are estimated to occur at the rate of $10^{-6}$ to $10^{-7}$ per gene replication, which can change several-fold under special conditions. ${ }^{24}$ Thus, host cell modifications of a random nature can result in the loss of productivity. ${ }^{4}$

Loss of productivity in a single cell assumes importance if the cell proliferates at a higher rate than the original strain. A higher proliferative status of nonproducing cells is likely due to the reduced productivity burden on cellular metabolism. Thus, the growth advantage of a nonproducing mutant represented by the ratio of growth rates determines the culture stability. In the present study we have investigated the burden that productivity places on cellular metabolism and the conditions of oxygenation that may help reduce this stress. Such conditions, favoring stability of the genetically engineered population, can be useful to obtain a large culture size for biochemical production.

The flux balance model coupled to a maximal growth objective provides a useful method for estimating culture stability. One assumes here the principle of stoichiometrically optimal metabolism. In a similar vein, the demand theory for genetic regulation predicts that the mechanisms of genetic control are determined by evolutionary optimality principles. Much theory and experimental data exist to provide a validation for the demand theory of genetic regulation. ${ }^{15-19}$

As a model for oxygen consumption by a cell we permit consumption up to an arbitrarily specified supply rate. Thus, by suitably specifying the oxygen supply rate we are able to determine metabolic behavior over the entire range of oxygen supply rates. It should be noted that although we only consider a variation in the oxygen supply, the key variable changed is the oxygen-to-glucose supply ratio.

The complete range of oxygenation has been investigated to determine the growth characteristics during biochemical production. We observe that depending on the product, an 
appropriate choice of oxygen supply can lead to a reduced growth advantage of nonproducer cells over the engineered high-producing strain. Since even a small reduction in the growth advantage can result in many more useful generations before the eventual outgrowth by nonproducers, a dramatic increase in the stability of the engineered strain can be obtained. Thus, the secretion of products from a cell can be stabilized through an appropriate selection of culture parameters.

For the illustrative examples of valine, tryptophan, and lysine production we demonstrate that the stability of an engineered strain can be markedly improved by an appropriate choice of oxygenation conditions. Based on economic criteria for production these improvements in culture stability can translate into large differences in the longevity of an economically viable culture. Thus, a culture stabilized by optimal oxygenation shows an improvement in the total production over several orders of magnitude.

Limitations of culture stability are an important consideration in the overall design of a bioprocess. In general, we find that the stability of a biochemical producing culture is very sensitive to the probability of mutation as well as the engineered productivity. However, significant improvements in stability can be obtained by an appropriate choice of oxygenation parameters. An investigation of culture parameters as a means for relieving production stress and thereby enhancing culture stability is therefore useful for the optimal design of a bioprocess.

The present study puts forth the important concept of controlling process parameters for improving the culture stability in a culture of genetically modified cells. Control of oxygen supply is an engineering technique that can be easily used to supplement other genetic techniques in order to enhance the stability of microbial cultures in bioprocesses. Thus, an integrated approach to bioprocess design using both strain development and process development to achieve the goals of high productivity and stability is expected to improve performance.

\section{NOMENCLATURE}

a mutational probability per cell division

$A$ ratio of mutational rate to growth rate of producing population

b vector representing transport flux of metabolites out of the cell

$C$ engineered conversion (mol product/mol substrate)

$C_{\max }$ maximum possible productivity (mol product/mol substrate)

$d_{M}$ metabolic demands for growth (mmol metabolite/g biomass)

$M$ a particular metabolite in the metabolic network

$n$ subscript that refers to the nonproducing population

$p$ subscript that refers to the producing population

$P$ fractional engineered conversion compared to the maximal conversion $\left(C / C_{\max }\right)$

$r_{s}$ rate of substrate uptake (mol/g DW-h)

$S$ growth advantage of a nonproducing population expressed as a ratio $\left(\mu_{n} / \mu_{p}\right)$

S stoichiometric matrix for the metabolic network; an element $S_{i j}$ represents the moles of metabolite $i$ needed for reaction $j$

$t$ time (h)

$t_{f}$ culture termination time (h)

TP total production (mmol product) $v$ vector of reaction fluxes (mmol flux/g DW-h); these fluxes are determined using linear optimization of an objective function

$v_{\text {gro }}$ biosynthetic growth flux defined as a drain of biosynthetic precursors in a ratio appropriate to produce cell biomass

$X$ cell mass (g DW)

$Y$ critical economic productivity (mol product/mol substrate)

$Z$ denotes the linear optimization objective

Greek letters

$\alpha$ mutational rate $\left(\mathrm{h}^{-1}\right)$

$\gamma_{i}$ shadow price of $i$ th metabolite

$\mu$ growth rate $\left(\mathrm{h}^{-1}\right)$

$\sigma$ dimensionless economic productivity $\left(Y / C_{\max }\right)$

$\tau$ dimensionless time $\left(\mu_{p} \cdot t\right)$

\section{References}

1. Anderson, K. B., von Meyenburg, K. 1980. Are growth rates of Escherichia coli in batch cultures limited by respiration? J. Bacteriol. 144: 114-123.

2. Bailey, J. E. 1991. Toward a science of metabolic engineering. Science 252: $1668-1675$.

3. Fell, D. A., Small, J. A. 1986. Fat synthesis in adipose tissue. An examination of stoichiometric constraints. Biochem. J. 238: 781-786.

4. Fu, J., Togna, A.P., Shuler, M.L., Wilson, D. B. 1992. Escherichia coli host cell modifications in continuous culture affecting heterologous protein overproduction: A population dynamics study. Biotechnol. Prog. 8: 340-346.

5. Gyr, M. 1978. Linear optimization using the simplex algorithm (simple). In CERN computer center program library. CERN, Geneva, Switzerland.

6. Imanaka, T., Aiba, S. 1981. A perspective on the application of genetic engineering: Stability of recombinant plasmid. Ann. NY Acad. Sci. 369: 1-14.

7. Ingraham, J. L., Maalæe, O., Neidhardt, F.C. 1983. Growth of the bacterial cell. Sinauer associates, Sunderland, MA.

8. Lee, G. M., Varma, A., Palsson, B. O. 1991. Application of population balance model to the loss of hybridoma antibody productivity. Biotechnol. Prog. 7: 72-75.

9. Majewski, R. A., Domach, M.M. 1990. Simple constrainedoptimization view of acetate overfiow in E. coli. Biotechnol. Bioeng. 35: 732-738.

10. Marr, A. G., Nilson, E.H., Clark, D.J. 1963. The maintenance requirement of Escherichia coli. Ann. N.Y. Acad. Sci. 102: 536-548.

11. Murty, K. G. 1983. Liner programming. Wiley, New York.

12. Neidhardt, F.C. 1987. Chemical composition of Escherichia coli. pp. 3-6 In: F. C. Neidhardt (ed.), Escherichia coli and Salmonella typhimurium. Cellular and molecular biology. American Society for Microbiology, Washington, DC.

13. Pirt, S.J. 1965. The maintenance energy of bacteria in growing cultures. Proc. Roy. Soc. Lond. Series B 163: 224-231.

14. Rubinow, S. I. 1975. Introduction to mathematical biology. Wiley, New York.

15. Savageau, M.A. 1974. Genetic regulatory mechanisms and the ecological niche of Escherichia coli. Proc. Natl. Acad. Sci. 71: 2435-2455.

16. Savageau, M.A. 1977. Design of molecular control mechanisms and the demand for gene expression. Proc. Natl. Acad. Sci. 74: 5647-5651.

17. Savageau, M.A. 1983. Regulation of differentiated cell-specific functions. Proc. Natl. Acad. Sci. 80: 1411-1415.

18. Savageau, M. A. 1985. Mathematics of organizationally complex systems. Biomed. Biochim. Acta 44: 839-844.

19. Savageau, M.A. 1985. A theory of alternate designs for biochemical control systems. Biomed. Biochim. Acta 44: 875-880.

20. Savinell, J. M., Palsson, B. O. 1992. Network analysis of intermediary metabolism using linear optimizations: I. Development of mathematical formalism. J. Theor. Biol. 154: 421-454. 
21. Schulze, K.L., Lipe, R.S. 1964. Relationship between substrate concentration, growth rate, and respiration rate of Escherichia coli in continuous culture. Arch. Microbiol. 48: 1-20.

22. Sonnleitner, B., Kappeli, O. 1986. Growth of Saccharomyces cerevisae is controlled by its limited respiratory capacity: Formulation and verification of a hypothesis. Biotechnol. Bioeng. 28: 927-937.

23. Stephanopoulos, G., Vallino, J. J. 1991. Network rigidity and metabolic engineering in metabolite overproduction. Science 252: 1675-1681.

24. Trobner, W., Piechocki, R. 1985. Selective advantage of polA1 mutator over polA + strains of Escherichia coli in a chemostat. Naturwissenschaften 72: 377-378.
25. Varma, A., Boesch, B. W., Palsson, B. O. 1993. Biochemical production capabilities of Escherichia coli Biotechnol. Bioneg. 42: 59-73.

26. Varma, A., Boesch, B.W., Palsson, B. O. 1993. Stoichiometric interpretation of Escherichia coli glucose catabolism under varying oxygenation rates. Appl. Environ. Microb. 59: 2465-2473.

27. Varma, A., Palsson, B. O. 1993. Metabolic capabilities of Escherichia coli: I. Synthesis of biosynthetic precursors and cofactors. J. Theor. Biol., to appear.

28. Varma, A., Palsson, B. O. 1993. Metabolic capabilities of Escherichia coli: II. Optimal growth patterns. J. Theor. Biol., to appear.

29. Watson, M.R. 1986. A discrete model of bacterial metabolism. Comput. Appl. Biosci. 2: 23-27. 